\title{
Inhibition of p38MAPK and CD137 signaling reduce dengue virus-induced TNF-a secretion and apoptosis
}

\author{
Amar Nagila1,2†, Janjuree Netsawang ${ }^{5+}$, Aroonroong Suttitheptumrong ${ }^{1,4}$, Atthapan Morchang ${ }^{1,4}$, \\ Sasiprapa Khunchai ${ }^{1,4}$, Chatchawan Srisawat, Chunya Puttikhunt ${ }^{6}$, Sansanee Noisakran ${ }^{6}$, \\ Pa-thai Yenchitsomanus ${ }^{1}$ and Thawornchai Limjindaporn ${ }^{1,3^{*}}$
}

\begin{abstract}
Background: Hepatic injury in dengue virus (DENV) infection is authenticated by hepatomegaly and an upsurge in transaminase levels. DENV replicates in hepatocytes and causes hepatocyte apoptosis both in vitro and in vivo. Understanding the molecular mechanisms of DENV-induced hepatic injury could facilitate the development of alternate chemotherapeutic agents and improved therapies.

Findings: The p38 mitogen-activated protein kinase (MAPK) participates in both apoptosis-related signaling and pro- inflammatory cytokine production. The role of p38 MAPK in DENV-infected HepG2 cells was examined using RNA interference. The results showed that DENV infection activated p38 MAPK and induced apoptosis. The p38 MAPK activation and TNF-a production were controlled by P38 MAPK and CD137 signaling in DENV-infected HepG2 cells as activated p38 MAPK, TNF-a and apoptosis were significantly decreased in p38 MAPK and CD137 depleted DENV-infected HepG2 cells. Addition of exogenous TNF-a to p38 MAPK depleted DENV-infected HepG2 cells restored DENV-induced apoptosis in HepG2 cells.
\end{abstract}

Conclusion: DENV induces CD137 signaling to enhance apoptosis by increasing TNF-a production via activation of p38 MAPK.

Keywords: Dengue virus, Apoptosis, TNF-a, p38MAPK, CD137

\section{Findings}

Hepatic dysfunction is a crucial feature of DENV infection. Hepatic biopsy specimens obtained from fatal cases of dengue shock syndrome (DSS) show cellular apoptosis, which may relate to the pathogenesis of DSS $[1,2]$. DENV replicates in hepatocytes and apoptosis of DENV-infected hepatic cells has been observed both in vitro and in vivo. Both the cell death receptor and the mitochondrial apoptotic pathways are affected [3-9].

DENV capsid protein (DENV C) activates both extrinsic and intrinsic apoptotic pathways in hepatic cell lines

\footnotetext{
* Correspondence: thawornchai.lim@mahidol.ac.th

${ }^{\dagger}$ Equal contributors

'Division of Molecular Medicine, Department of Research and Development, Faculty of Medicine Siriraj Hospital, Mahidol University, Bangkok, Thailand ${ }^{3}$ Department of Anatomy, Faculty of Medicine Siriraj Hospital, Mahidol University, Bangkok, Thailand

Full list of author information is available at the end of the article
}

[10-12]. DENV C localizes to both the cytoplasm and nucleus of DENV-infected HepG2 cells. DENV C contains three nuclear localization signals (NLS), ${ }^{6} \mathrm{KKAR}^{9}$, ${ }^{73} \mathrm{KKSK}^{76}$ and the bipartite signal ${ }^{85}$ RKeigrmlnilnRRRR ${ }^{100}$. Substitution mutations in DENV C (R85A/K86A) result in loss of nuclear localization, Daxx interaction, and apoptosis [11]. Comparison of the apoptotic gene expression profile of DENV C and DENV C (R85A/K86A) transfected HepG2 cells showed a significant increase in expression of CD137, a member of TNF receptor family, in HepG2 cells expressing DENV C. In DENV-infected HepG2 cells, CD137 and CD137 ligand mRNA expression increased 60-fold and 3-fold post DENV infection, respectively. CD137 positive cells increased, but less dramatically, about 3-fold post infection and correlates with the level of apoptosis induced by DENV infection of HepG2 cells, which increased 4-fold post DENV infection [13].

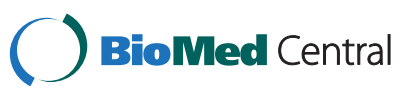


CD137 recruits TNF receptor associated factor 2 (TRAF2) and activates apoptosis signal regulating kinase 1 (ASK1), resulting in activation of cJun N-terminal kinase (JNK) and p38 mitogen-activated protein kinase (MAPK) [14]. p38 MAPK is primarily implicated in apoptosisrelated signaling [15]. However, activation of p38 MAPK increases inflammatory cytokine production [16]. Activation of p38 MAPK may contribute to the pathogenesis of DENV-induced apoptosis via both mechanisms.

\section{Inhibition of DENV-induced apoptosis in HepG2 cells by siRNA against p38 MAPK}

We first asked whether RNAi knockdown of p38 $\alpha$ MAPK, which is a crucial mediator of proinflammatory cytokine production, would inhibit DENV-induced apoptosis in HepG2 cells, an approach that might alleviate DENVmediated hepatic injury. At $24 \mathrm{~h}$ after seeding, HepG2 cells were transfected with 200 pmol of siRNA directed against p38 $\alpha$ MAPK, 5'CAGACCATATTAACCAGCTTC AGCA3' or with siControl, 5' CACGCCTCTTTGTCTT GTTTCGAAA 3' (Invitrogen), using lipofectamine 2000 (Invitrogen). Twenty-four hours post transfection, the cells were infected with DENV serotype 2 strain 16681 at an MOI of 5 using the methods described previously [13]. The percentage of apoptotic cells was determined by annexin V/FITC and PI double staining (BD Biosciences) and quantitation by flow cytometry. The efficiency of p38 $\alpha$ MAPK knockdown by siRNA was examined by realtime RT-PCR using p38 $\alpha$ MAPK-specific primers (p38 $\alpha$ MAPK-F, 5'CGACTTGCTGGAGAAGATGC $3^{\prime}$, and p38 $\alpha$ MAPK-R, 5'TCCATCTCTTCTTGGTCAAGG3') and by Western blot analysis using primary antibodies against p38 MAPK and $\beta$-actin (Santa Cruz Biotechnology). About $30 \%$ decreases in both mRNA and protein expression levels of p38 MAPK were observed (Figure 1A, 1B). In addition, treatment with siRNA against p38 MAPK
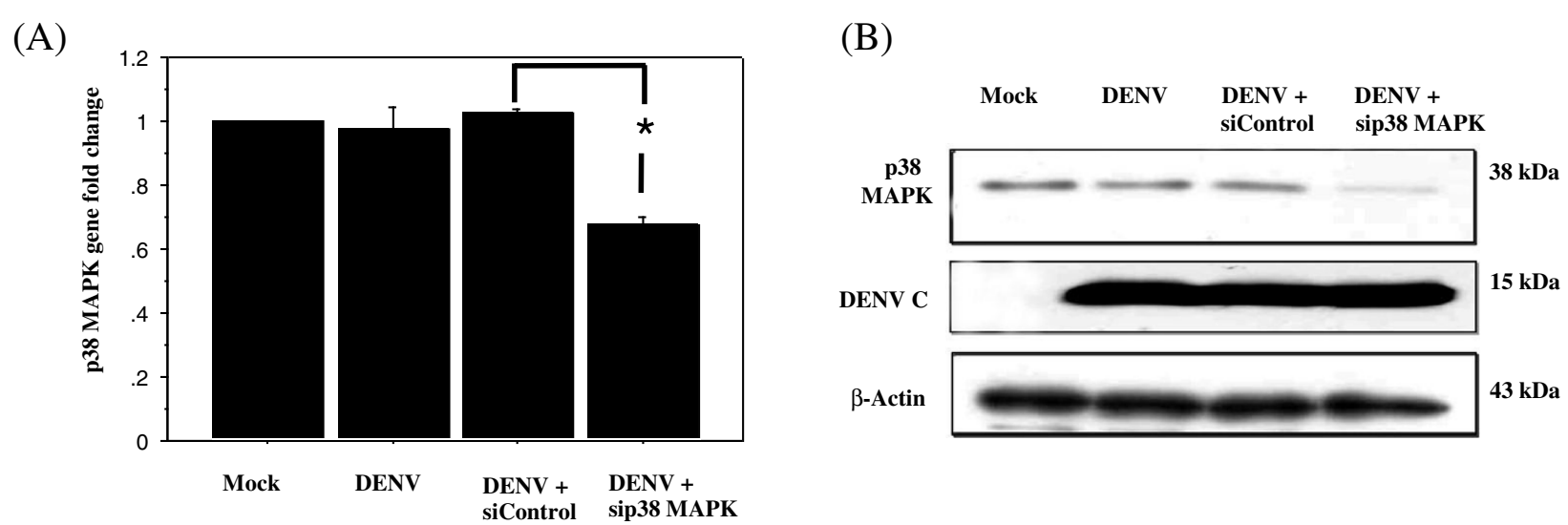

(C)
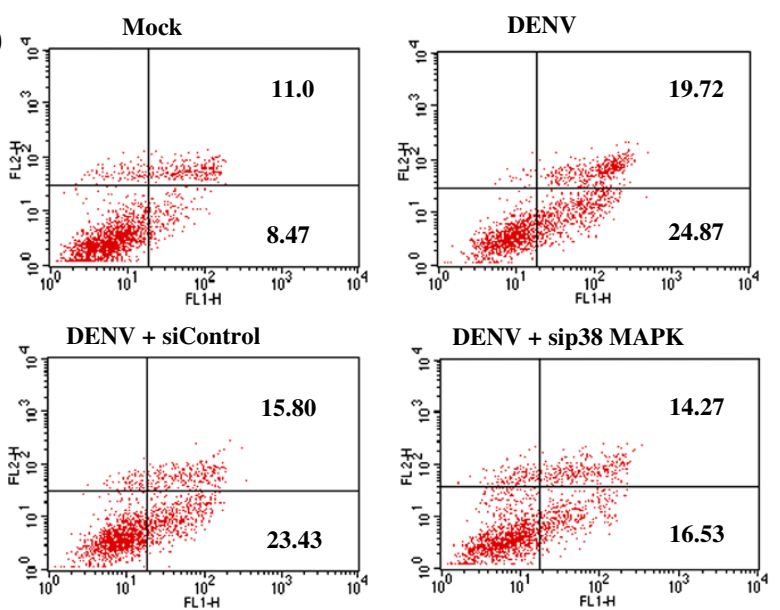

(D)

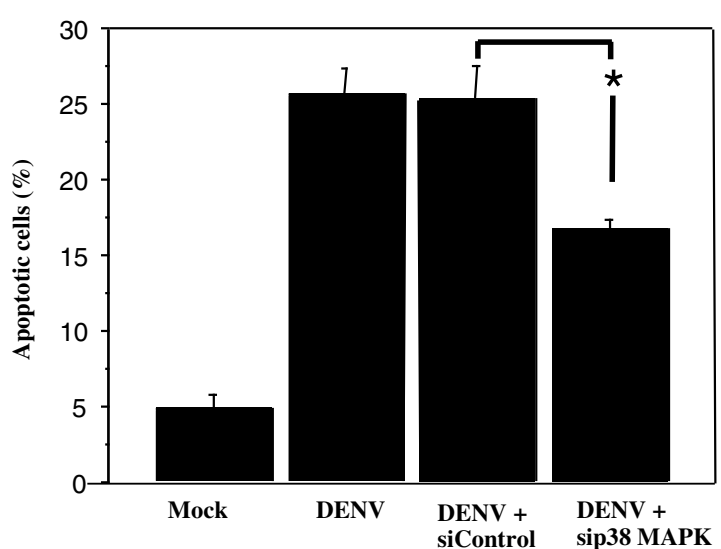

Figure 1 Decreased apoptosis in p38 MAPK knockdown DENV-infected HepG2 cells. HepG2 cells were transfected either with siRNA directed against p38 MAPK or with control siRNA. Twenty-four hours post transfection, cells were infected with DENV at MOI 5 for $60 \mathrm{~h}$. Cells were harvested and analyzed for (A) mRNA level of p38 MAPK (B) protein level of p38 MAPK. (C) Apoptosis by flow cytometry. Apoptosis cells have annexin V+/PI- staining. (D) Bar graph represents apoptosis experiments. All data were obtained from three independent experiments and reported as the mean \pm SEM. Statistical differences between the groups were tested with an unpaired $t$-test using StatView version 5.0 and $P$ value less than 0.05 was considered significant. 
reduced apoptosis from $23.43 \%$ to $16.53 \%$ in DENVinfected HepG2 cells (Figure 1C, D) suggesting a role for p38 MAPK in DENV-mediated apoptosis. The result of siRNA directed against p38 MAPK in DENV-infected HepG2 cells is agreeable to our previous study of pharmacological inhibition of p38 MAPK by SB203580 in DENVinfected HepG2 cells [17]. Therefore, genetic inhibition of p38 activity reproduces pharmacological inhibition of the enzyme and confirms the contribution of p38 MAPK to apoptotic events induced by DENV [17]. DENV infected HepG2 cells equally in the presence or absence of SB203580 [17] but DENV production in BHK-21 cells was decreased in the presence of SB203580 [18].

Inhibition of DENV C-induced apoptosis in stable HepG2 cell expressing DENV $C$ by siRNA against p38 MAPK

We next asked whether DENV C activates p38 MAPKinduced apoptosis by examining the effect of siRNA against p38 $\alpha$ MAPK on apoptosis of HepG2 cells stably expressing DENV C. HepG2 cells stably expressing DENV $C$ were constructed in this study using retroviral system (Stratagene). Briefly, 70\% confluent of HEK $293 \mathrm{~T}$ cells was transfected with p-VPack-GP, p-VPackVSV-G and pFB-Neo-DENV C using lipofectamine 2000 (Invitrogen). The culture supernatant containing infectious viral particles was collected after $24 \mathrm{~h}$ post transfection and added to HepG2 cells, which were preincubated with $8 \mu \mathrm{g} / \mathrm{ml}$ of polybrene. Twenty-four h after incubation, stable HepG2 cells expressing DENV C were selected with media containing $0.5 \mathrm{mg} / \mathrm{ml} \mathrm{G418}$ (Calbiochem). The G418-resistant cells were grown and maintained in DMEM medium containing $0.5 \mathrm{mg} / \mathrm{ml}$ G418, and the expression of DENV C was examined by flow cytometry and Western blot analysis using antibody to DENV C [19]. Up to $5 \times 10^{5}$ the stably expressing cells were plated for $24 \mathrm{~h}$ prior to transfection. The cells were then transfected with siRNAs as described in the preceding experiment. Knock-down efficiency was assessed by Western blot analysis. The $15 \mathrm{kDa}$ capsid protein was expressed in stable HepG2 cells expressing DENV but not in HepG2 cells expressing control plasmid (Figure 2A). To activate the extrinsic apoptotic

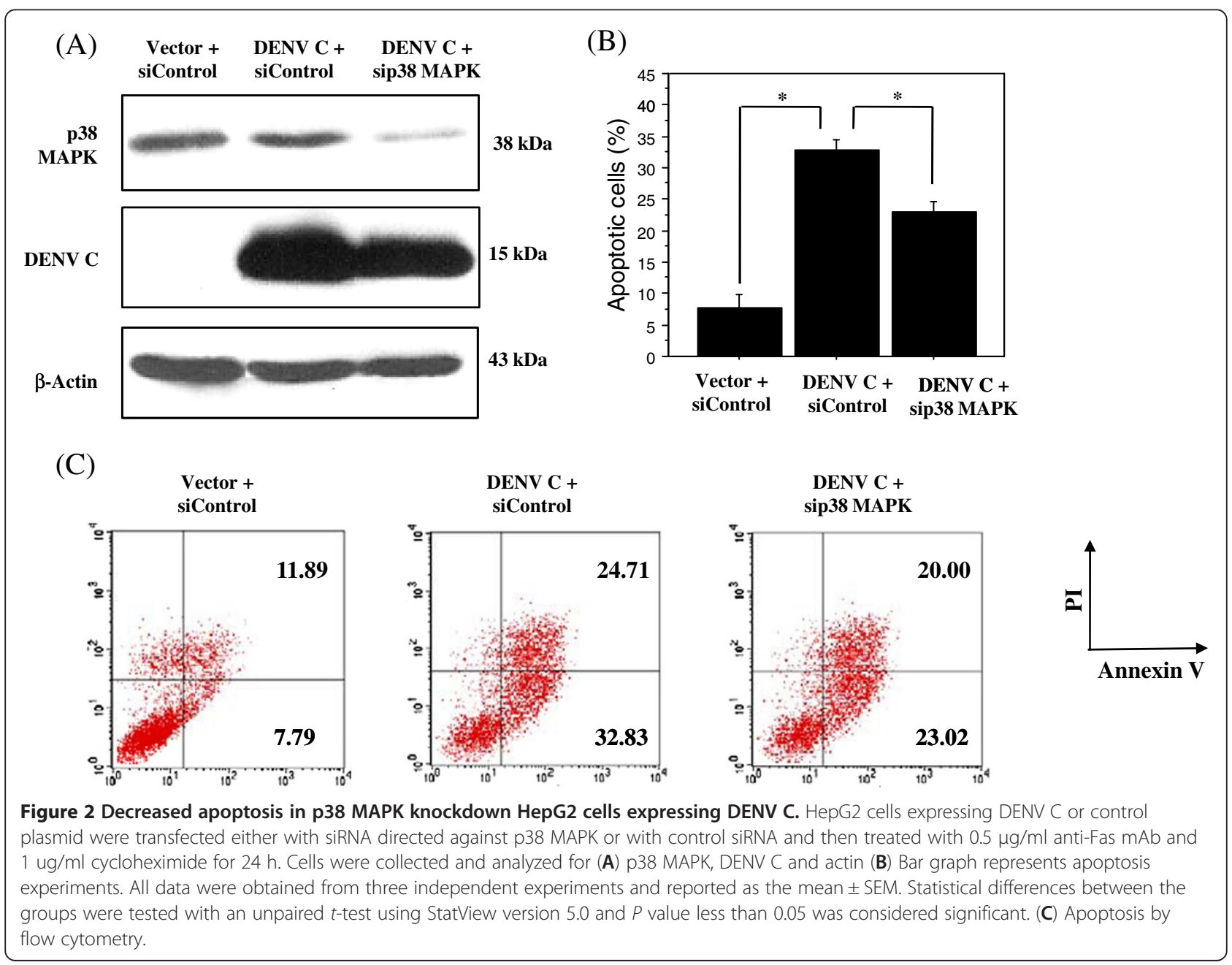


pathway, cells were treated with $0.5 \mu \mathrm{g} / \mathrm{ml}$ anti-Fas $\mathrm{mAb}$ (Sigma) and $1 \mathrm{ug} / \mathrm{ml}$ cycloheximide (Sigma) for $24 \mathrm{~h}$ in culture medium. Both adherent and floating cells, as well as culture supernatants, were collected and assessed for apoptosis by annexinV/PI staining (BD Biosciences). As with DENV-infected cells, siRNA p38 $\alpha$ MAPK resulted in a substantial reduction in $\mathrm{p} 38$ MAPK protein, but had no effect on DENV C expression (Figure 2A). In the presence of anti-Fas and cycloheximide, apoptosis of HepG2 cells expressing DENV C increased from $7.79 \%$ to $32.83 \%$ compared to that of HepG2 cells expressing control plasmid. HepG2 cells expressing DENV C transfected with p38 MAPK siRNA reduced apoptosis from $32.83 \%$ to $23 \%$ (Figure 2B, 2C). In our previous study without anti-Fas $\mathrm{mAb}$ and cycloheximide treatment, HepG2 cells were transiently transfected with a DENV C or control plasmid and incubated in the presence of DMSO or $10 \mu \mathrm{M}$ of SB203580 for $24 \mathrm{~h}$. The percentage of apoptotic cells was then determined by annexin V/FITC and PI double staining and quantitation by flow cytometry. Comparable to apoptosis of HepG2 cells expressing control plasmid, apoptosis of HepG2 cells expressing DENV C increased from $10.11 \%$ to $24.40 \%$. Apoptosis of HepG2 cells expressing DENV C decreased from $24.40 \%$ to $5.69 \%$ in medium with SB203580 [17]. Therefore, genetic inhibition of p38 activity reproduces pharmacological inhibition of the enzyme and verifies the contribution of p38 MAPK to apoptotic events induced specifically by DENV C. However, not all DENV-induced cell death is caused by DENV $\mathrm{C}$, other DENV proteins including M, NS3 protease and NS2B-NS3 precursor also induces apoptosis $[20,21]$.

\section{Inhibition of DENV-induced phosphorylated p38 MAPK, TNF-a production and apoptosis in HepG2 cells by siRNA against CD137}

To further define the molecular mechanisms involved, we asked whether CD137 signaling regulates p38 MAPK activation and apoptosis in DENV-infected HepG2 cells. siRNA knockdown of CD137 was performed as described for p38 MAPK using the CD137-specific oligo $5^{\prime}$ CACGCTCCGTTTCTCTGTTGTTAAA 3' (Invitrogen). The efficiency of knockdown was examined by real-time RT-PCR using CD137-specific primers CD137-F, 5'CCA AAA TGT TCT GCT GAT CG3' ${ }^{\prime}$ and CD137-R, $5^{\prime}$ AAG ACT GTG GCG CCC TG3'. The number of CD 137 positive cells was measured by flow cytometry using a primary antibody against CD137 (Santa Cruz Biotechnology). Transfection of HepG2 cells with siRNA against CD137 resulted in a nearly 2-fold reduction in CD137 mRNA and CD137-positive cells (Figure 3A, 3B). The effect of CD137 depletion on p38 MAPK activation during DENV infection and apoptosis were measured by Western blot analysis using primary antibody against phosphorylated p38 MAPK (Santa Cruz Biotechnology), and by annexinV/
PI staining (BD Biosciences), respectively. Knockdown of CD137 expression reduced the amount of phosphorylated p38 MAPK (Figure 3C) and apoptosis (Figure 3D). These results indicate a role of CD137 signaling in regulation of p38 MAPK activation and apoptosis in DENV-infected HepG2 cells. As DENV induced CD137 expression only $30 \%$ of the infected cells (Figure $3 \mathrm{~B}$ ) and expressed at the late stage of infection [13], CD137 and p38 MAPK signaling may support other apoptotic signaling pathways [3-9] to aggravate apoptosis at the late stage of DENV infection. Multiple studies in vitro and in vivo models implicate TNF- $\alpha$ in DENV-induced tissue damage [8,22]. In addition, TNF- $\alpha$ induced apoptosis via p38 MAPK activation was shown in pseudorabies virus infection [23].

We next asked whether TNF- $\alpha$ mediated DENVinduced apoptosis via CD137 signaling. TNF- $\alpha$ expression in CD137 depleted DENV-infected HepG2 cells was examined by real-time RT-PCR using TNF- $\alpha$-specific primers TNF- $\alpha-F, 5^{\prime}$ TGCTTGTTCCTCAGCCTCTT $3^{\prime}$, and TNF- $\alpha-R, 5^{\prime}$ ATGGGCTACAGGCTTGTCACT3' and by ELISA (R\&D Systems). DENV infection of HepG2 cells resulted in a dramatic increase in TNF- $\alpha$ mRNA and protein (Figure 3E, 3F). However, CD137 siRNA treatment reduced of TNF- $\alpha$ production about 30\% (Figure 3E, 3F), which correlates well with the comparable reduction in apoptosis of CD137 knockdown cells (Figure 3D).

\section{Inhibition of p38 MAPK activity by SB203580 decreased TNF-a production in DENV-infected HepG2 cells}

As p38 MAPK is one of the downstream targets of CD137 signaling, it is important to assess whether inhibition of p38 MAPK activity in DENV-infected HepG2 cells would reduce TNF- $\alpha$ expression. HepG2 cells were infected with DENV serotype 2 at a MOI 5 and incubated $48 \mathrm{~h}$ in the presence of either DMSO or $10 \mu \mathrm{M}$ SB203580 (Santa Cruz Biotechnology). TNF- $\alpha$ mRNA expression of DENVinfected HepG2 cells and TNF- $\alpha$ protein expression in supernatant of DENV-infected HepG2 cells were subsequently examined by real-time RT-PCR and by ELISA, respectively. As expected, treatment with $10 \mu \mathrm{M}$ SB203580 significantly reduced TNF- $\alpha$ expression in DENV-infected HepG2 cells (Figure 4A, 4B) indicating that pharmacological inhibition of p38 MAPK reduced TNF- $\alpha$ secretion in DENV-infected HepG2 cells. Whether inhibition of both p38 and CD137 signalings has the additive effect on TNF- $\alpha$ production merits further investigation.

\section{The role of TNF-a expression in apoptosis of DENV- infected HepG2 cells}

It would be important to evaluate the effect of TNF- $\alpha$ expression in apoptosis of DENV-infected HepG2 cells. Supernatants containing DENV from mock-infected or DENV-infected HepG2 cells in the presence of either 


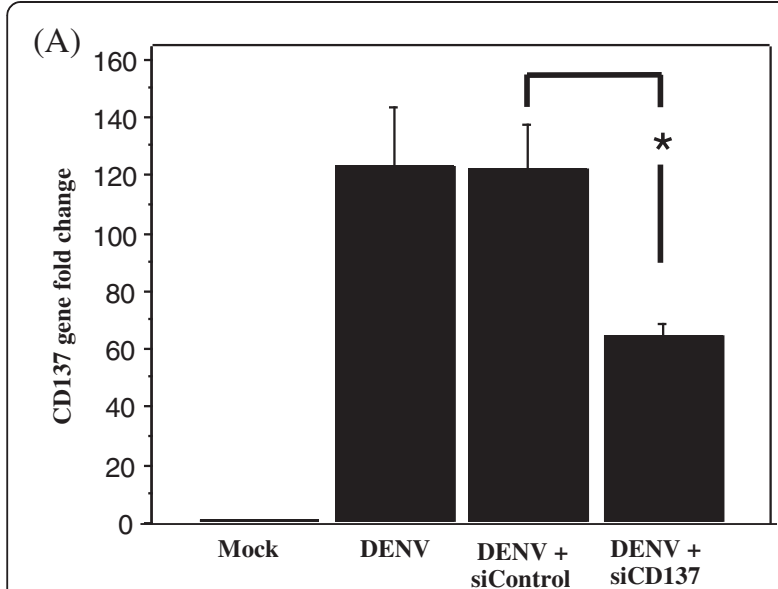

(C)

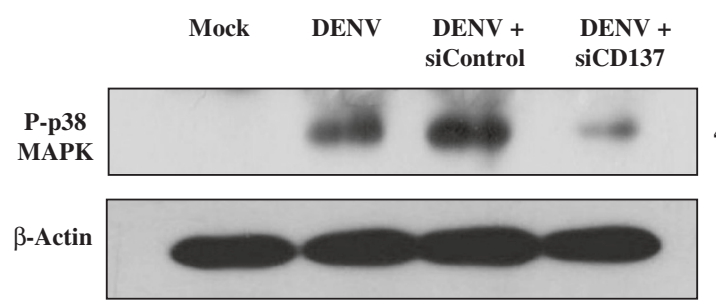

(E)

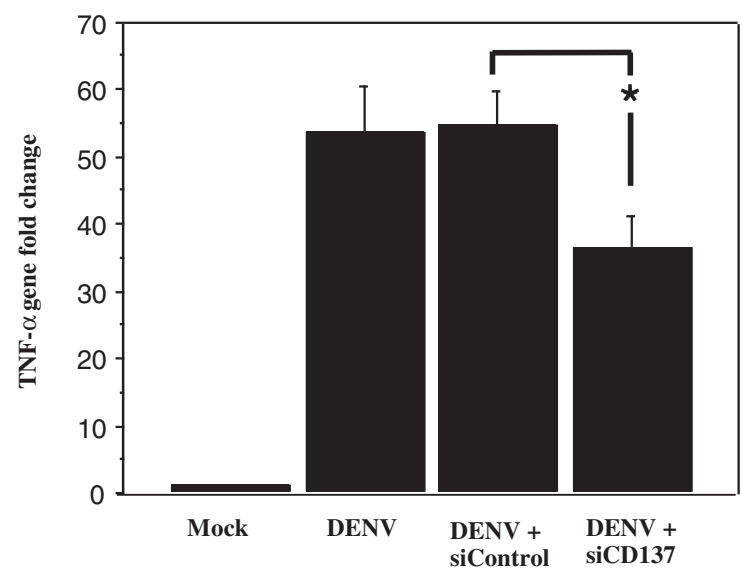

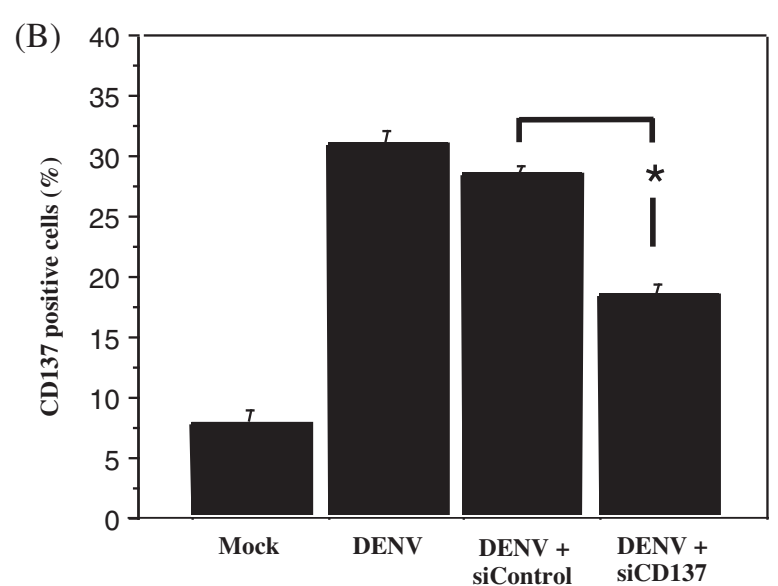

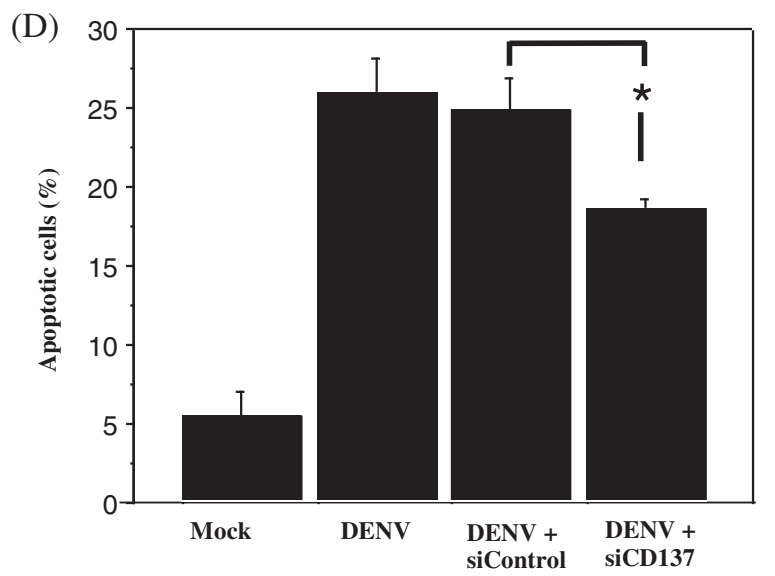

(F)

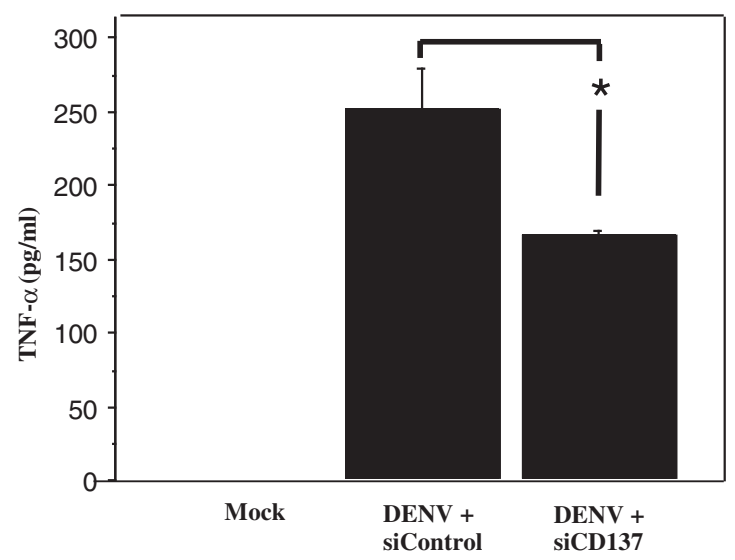

Figure 3 Reduced DENV-induced phosphorylated p38 MAPK, TNF-a and apoptosis in HepG2 cells by siRNA against CD137. HepG2 cells were transfected with either CD137 siRNA or control siRNA. Twenty-four hours post transfection, cells were infected with DENV at MOI 5 for $60 \mathrm{~h}$. Cells were collected and analyzed for (A) CD137 mRNA (B) CD137 positive cells (C) phosphorylation of p38 and (D) Apoptosis by flow cytometry. The cells were collected and analyzed for TNF-a mRNA (E) and the culture supernatant was analyzed for TNF-a protein (F). All data were obtained from three independent experiments and reported as the mean \pm SEM. Statistical differences between the groups were tested with an unpaired $t$-test using StatView version 5.0 and $P$ value less than 0.05 was considered significant. 


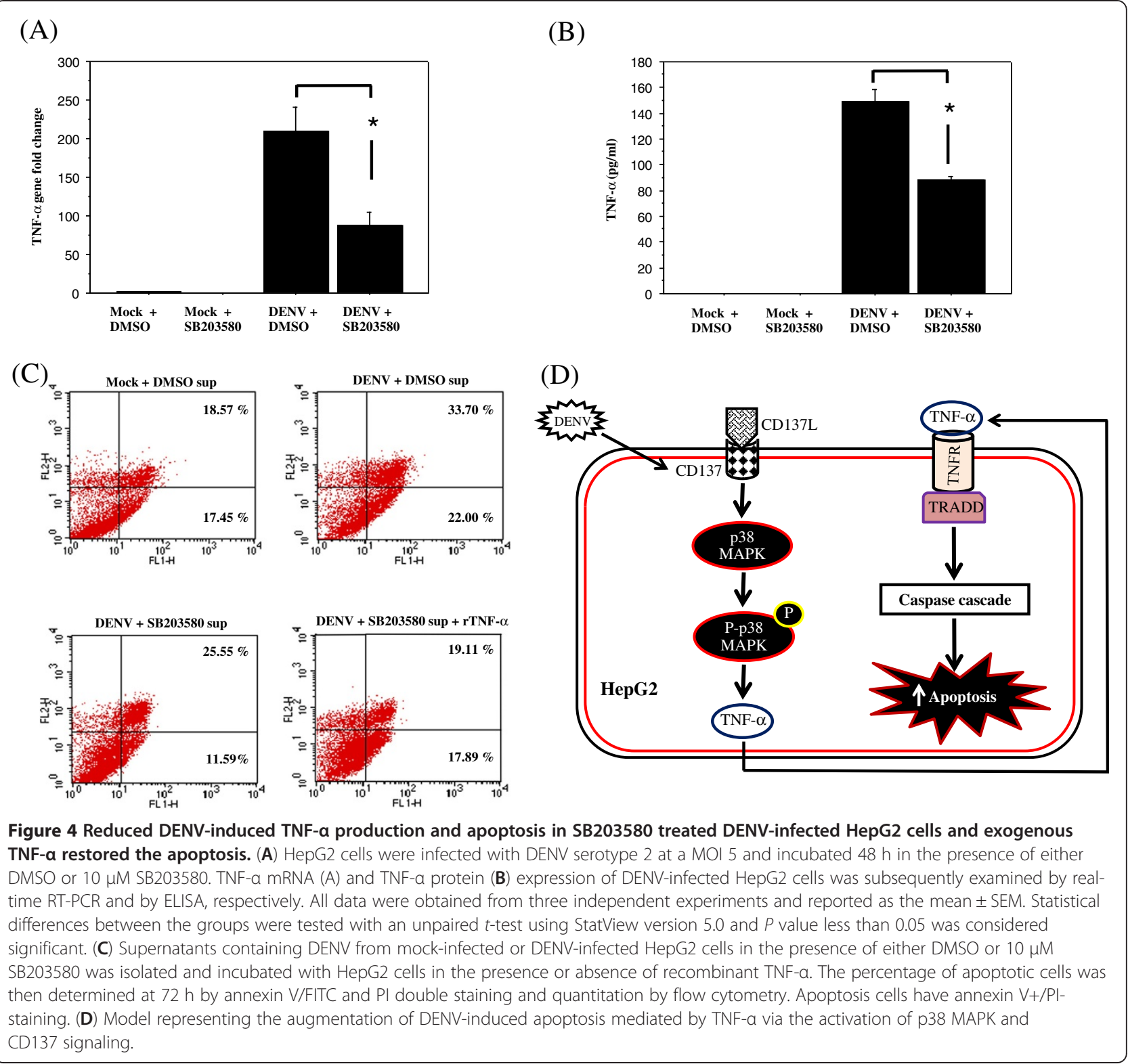

DMSO or $10 \mu \mathrm{M}$ SB203580 was isolated and further incubated with HepG2 cells in the presence or absence of recombinant TNF- $\alpha$ (250 ng/ml)(Sigma). The percentage of apoptotic cells was then determined at $72 \mathrm{~h}$ by annexin V/FITC and PI double staining and quantitation by flow cytometry. The result in Figure 4C showed that apoptosis of DENV-infected HepG2 cells decreased from $22 \%$ to $11.59 \%$ when HepG2 cells were incubated with supernatant containing virus from SB203580 treated DENV-infected HepG2 cells. Addition of recombinant TNF- $\alpha$ to supernatant from SB203580 treated DENV-infected HepG2 cells increased apoptosis of DENV-infected HepG2 cells from $11.59 \%$ to $17.89 \%$ (Figure $4 \mathrm{C}$ ) suggesting that TNF- $\alpha$ production affects DENV-induced apoptosis of HepG2 cells. Our result supports the previous studies for the role of TNF- $\alpha$ expression in DENV-induced apoptosis in other cell lines. TNF- $\alpha$ produced from macrophage was shown to enhance DENV-induced endothelial cell death [24] and inhibition of peripheral blood mononuclear cell (PBMC) apoptosis by etanercept, which is the antibody to TNF- $\alpha$, was also reported [25]. In summary, we propose in Figure 4D that DENV induces phosphorylated p38 MAPK to stimulate apoptosis and inhibition of $\mathrm{p} 38$ MAPK and CD137 pathway reduce DENV-induced TNF- $\alpha$ secretion and apoptosis of HepG2 cells.

\section{Competing interests}

The authors declare that they have no competing interest. 


\section{Authors' contributions}

$T L, P Y$ and SN conceived of the study. AN, JN, AM and CS carried out experiments in RNA interference and apoptosis. AS and SK carried out cytokine assay. CP participated in antibody production. All authors have read and approved the final version of the manuscript.

\section{Acknowledgements}

We appreciate Dr. William A Fonzi, Georgetown University, USA, for the critical reading and editing of this manuscript. This work was financially supported by Mahidol University Grant and Siriraj Research Developmental Fund to T.L., who is a Mid-Career Thailand Research Fund-Commission of Higher Education (TRF-CHE) Research Scholar. PY is a TRF-Senior Research Scholar. JN is a TRF-CHE Young Research Scholar. AM was supported by TRFRoyal Golden Jubilee (TRF-RGJ) Ph.D. Program. SK was supported by CHE under the program strategic scholarship for frontier research network for the join Ph.D. program. AS was supported by Siriraj Developmental Scholarship and AN was supported by Siriraj Graduate Thesis Scholarship. All authors obtained permission to acknowledge the source of funding.

\section{Author details}

'Division of Molecular Medicine, Department of Research and Development, Faculty of Medicine Siriraj Hospital, Mahidol University, Bangkok, Thailand. ${ }^{2}$ Department of Biochemistry, Faculty of Medicine Siriraj Hospital, Mahidol University, Bangkok, Thailand. ${ }^{3}$ Department of Anatomy, Faculty of Medicine Siriraj Hospital, Mahidol University, Bangkok, Thailand. ${ }^{4}$ Department of Immunology, Faculty of Medicine Siriraj Hospital, Mahidol University, Bangkok, Thailand. ${ }^{5}$ Faculty of Medical Technology, Rangsit University, Bangkok, Thailand. ${ }^{6}$ Medical Biotechnology Research Unit, National Center for Genetic Engineering and Biotechnology, National Science and Technology Development Agency, Bangkok, Thailand.

Received: 14 December 2012 Accepted: 2 April 2013

Published: 4 April 2013

\section{References}

1. Bhamarapravati N: Hemostatic defects in dengue hemorrhagic fever. Rev Infect Dis 1989, 11(Suppl 4):S826-S829.

2. Limonta D, Capo V, Torres G, Perez AB, Guzman MG: Apoptosis in tissues from fatal dengue shock syndrome. J Clin Virol 2007, 40:50-54

3. Marianneau P, Cardona A, Edelman L, Deubel V, Despres P: Dengue virus replication in human hepatoma cells activates NF-kappaB which in turn induces apoptotic cell death. J Virol 1997, 71:3244-3249.

4. Thongtan T, Panyim S, Smith DR: Apoptosis in dengue virus infected liver cell lines HepG2 and Hep3B. J Med Virol 2004, 72:436-444.

5. Nasirudeen AM, Liu DX: Gene expression profiling by microarray analysis reveals an important role for caspase- 1 in dengue virus-induced p53mediated apoptosis. J Med Virol 2009, 81:1069-1081.

6. El-Bacha T, Midlej V, da Silva Pereira AP, da Costa Silva L, Benchimol M Galina A, Da Poian AT: Mitochondrial and bioenergetic dysfunction in human hepatic cells infected with dengue 2 virus. Biochim Biophys Acta 2007, 1772:1158-1166.

7. Matsuda T, Almasan A, Tomita M, Tamaki K, Saito M, Tadano M, Yagita H, Ohta T, Mori N: Dengue virus-induced apoptosis in hepatic cells is partly mediated by Apo2 ligand/tumour necrosis factor-related apoptosisinducing ligand. J Gen Virol 2005, 86:1055-1065.

8. Wati S, Rawlinson SM, Ivanov RA, Dorstyn L, Beard MR, Jans DA, Pitson SM, Burrell CJ, Li P, Carr JM: Tumour necrosis factor alpha (TNF-\{alpha\}) stimulation of cells with established dengue virus type 2 infection induces cell death that is accompanied by a reduced ability of TNF\{alpha\} to activate nuclear factor $\{k a p p a\} B$ and reduced sphingosine kinase-1 activity. J Gen Virol 2011, 92:807-818.

9. Liao $\mathrm{H}, \mathrm{Xu}$ J, Huang J: FasL/Fas pathway is involved in dengue virus induced apoptosis of the vascular endothelial cells. J Med Virol 2010 82:1392-1399

10. Limjindaporn T, Netsawang J, Noisakran S, Thiemmeca S, Wongwiwat W Sudsaward S, Avirutnan P, Puttikhunt C, Kasinrerk W, Sriburi R, et al: Sensitization to Fas-mediated apoptosis by dengue virus capsid protein. Biochem Biophys Res Commun 2007, 362:334-339.

11. Netsawang J, Noisakran S, Puttikhunt C, Kasinrerk W, Wongwiwat W, Malasit P, Yenchitsomanus PT, Limjindaporn T: Nuclear localization of dengue virus capsid protein is required for DAXX interaction and apoptosis. Virus Res 2010, 147:275-283.

12. Nasirudeen AM, Wang L, Liu DX: Induction of p53-dependent and mitochondria-mediated cell death pathway by dengue virus infection of human and animal cells. Microbes Infect 2008, 10:1124-1132.

13. Nagila A, Netsawang J, Srisawat C, Noisakran S, Morchang A, Yasamut U, Puttikhunt C, Kasinrerk W, Malasit P, Yenchitsomanus PT, Limjindaporn T: Role of CD137 signaling in dengue virus-mediated apoptosis. Biochem Biophys Res Commun 2011, 410:428-433.

14. Sollner L, Shaqireen DOKMM, Wu JT, Schwarz H: Signal transduction mechanisms of CD137 ligand in human monocytes. Cell Signal 2007, 19:1899-1908

15. Xia Z, Dickens M, Raingeaud J, Davis RJ, Greenberg ME: Opposing effects of ERK and JNK-p38 MAP kinases on apoptosis. Science 1995, 270:1326-1331.

16. Ono K, Han J: The p38 signal transduction pathway: activation and function. Cell Signal 2000, 12:1-13.

17. Morchang A, Yasamut U, Netsawang J, Noisakran S, Wongwiwat W, Songprakhon P, Srisawat C, Puttikhunt C, Kasinrerk W, Malasit P, et al: Cell death gene expression profile: role of RIPK2 in dengue virus-mediated apoptosis. Virus Res 2011, 156:25-34.

18. Ceballos-Olvera I, Chavez-Salinas S, Medina F, Ludert JE, del Angel RM: JNK phosphorylation, induced during dengue virus infection, is important for viral infection and requires the presence of cholesterol. Virology 2009, 396:30-36.

19. Puttikhunt C, Ong-Ajchaowlerd P, Prommool T, Sangiambut S, Netsawang J, Limjindaporn T, Malasit P, Kasinrerk W: Production and characterization of anti-dengue capsid antibodies suggesting the $\mathrm{N}$ terminus region covering the first 20 amino acids of dengue virus capsid protein is predominantly immunogenic in mice. Arch Virol 2009, 154:1211-1221.

20. Catteau A, Kalinina O, Wagner MC, Deubel V, Courageot MP, Despres P: Dengue virus $M$ protein contains a proapoptotic sequence referred to as ApoptoM. J Gen Virol 2003, 84:2781-2793.

21. Shafee N, AbuBakar S: Dengue virus type 2 NS3 protease and NS2B-NS3 protease precursor induce apoptosis. J Gen Virol 2003, 84:2191-2195.

22. Yen YT, Chen HC, Lin YD, Shieh CC, Wu-Hsieh BA: Enhancement by tumor necrosis factor alpha of dengue virus-induced endothelial cell production of reactive nitrogen and oxygen species is key to hemorrhage development. J Virol 2008, 82:12312-12324.

23. Yeh CJ, Lin PY, Liao MH, Liu HJ, Lee JW, Chiu SJ, Hsu HY, Shih WL: TNFalpha mediates pseudorabies virus-induced apoptosis via the activation of p38 MAPK and JNK/SAPK signaling. Virology 2008, 381:55-66.

24. Chen HC, Hofman FM, Kung JT, Lin YD, Wu-Hsieh BA: Both virus and tumor necrosis factor alpha are critical for endothelium damage in a mouse model of dengue virus-induced hemorrhage. J Virol 2007, 81:5518-5526.

25. Jaiyen $Y$, Masrinoul P, Kalayanarooj S, Pulmanausahakul R, Ubol S: Characteristics of dengue virus-infected peripheral blood mononuclear cell death that correlates with the severity of illness. Microbiol Immunol 2009, 53:442-450.

doi:10.1186/1743-422X-10-105

Cite this article as: Nagila et al:: Inhibition of p38MAPK and CD137 signaling reduce dengue virus-induced TNF-a secretion and apoptosis. Virology Journal 2013 10:105.

\section{Submit your next manuscript to BioMed Central and take full advantage of:}

- Convenient online submission

- Thorough peer review

- No space constraints or color figure charges

- Immediate publication on acceptance

- Inclusion in PubMed, CAS, Scopus and Google Scholar

- Research which is freely available for redistribution 